\title{
Air-suspended two-dimensional polymer photonic crystal slab waveguides fabricated by nanoimprint lithography
}

\author{
Choon-Gi Choi, ${ }^{\text {a) }}$ Young-Tak Han, and Jin Tae Kim \\ IT Convergence and Components Laboratory (ICCL), Electronics and Telecommunications Research \\ Institute (ETRI), Daejeon 305-700, Korea \\ Helmut Schift \\ Laboratory of Micro and Nanotechnology, Paul Scherrer Institut (PSI), 5232 Villigen PSI, Switzerland
}

(Received 11 January 2007; accepted 8 May 2007; published online 30 May 2007)

\begin{abstract}
Air-suspended membrane-type photonic crystals were fabricated in polystyrene. The two-dimensional photonic crystal slab nanostructures were replicated by a thermal nanoimprint process, and then air-suspended membrane structures were generated by a selective sacrificial layer etch process. The optical transmission showed clear band gap characteristics at $1550 \mathrm{~nm}$ wavelength, which is in good agreement with the theoretical calculations. () 2007 American Institute of Physics. [DOI: 10.1063/1.2744482]
\end{abstract}

Photonic crystals (PhCs) are periodic dielectric structures with refractive index contrast. The periodic dielectric structures exhibit interesting properties such as a photonic band gap (PBG) where the propagation of photons within a certain range of frequencies is forbidden. ${ }^{1}$ By incorporating structural defects such as point or line defects into PhC slabs, various functional nanophotonic devices can be implemented. ${ }^{2}$ Thus they are expected to serve as ultracompact photonic crystal integrated circuits (PCICs), which are the counterpart of integrated electronic circuits. $\mathrm{PhC}$ devices with one-dimensional, two-dimensional (2D), and threedimensional (3D) structures have been fabricated by wet/dry etching, wafer bonding, self-assembled method, and electron-beam lithography. ${ }^{3}$ Among these three types of structures, 2D PhC can be exploited to realize microcavity lasers and linear waveguides to direct the electromagnetic waves towards specific areas, thus allowing high integration in planar PCIC.,

Nanoimprint lithography (NIL) is now standing in the spotlight as an emerging nanofabrication technology for next-generation lithography because of its ability to produce nanoscale features with high throughput in a cost-effective way. ${ }^{6}$ Polymers have attracted great interest, due to their low temperature fabrication, good mass production possibilities with low processing cost, easy functionalization, and possibility to tune their optical properties. ${ }^{7,8}$ Compared to semiconductor materials such as InP, GaAs, and silicon on insulator (SOI), which are used for high refractive index contrast based $\mathrm{PhC}$, the low refractive index contrast based polymer PhC can allow a larger optical mode volume. 9

Recently, we showed that complete band gaps and line defect modes for TE-like polarizations can exist in the polymer photonic crystal slabs (PPhCSs) suspended in air with a triangular array of air holes. ${ }^{10}$ However, the PPhCSs with a triangular array of air holes were not implemented yet in slabs suspended in air. The specific challenges in the processing lie in the molding and demolding of dense pillar structures and in the residual layer etch with high structural fidelity, and also in the release of large polymer membranes from

${ }^{\text {a) }}$ Author to whom correspondence should be addressed; electronic mail: cgchoi@etri.re.kr a substrate without sagging. Different sacrificial layer methods were developed, among those using $\mathrm{SiO}_{2}$ and lift-off resists for controlled underetching and release. ${ }^{7,11}$

In this letter we report air-suspended membrane-type two-dimensional PPhCS waveguides fabricated by nanoimprint lithography. They have line defect waveguides with a triangular array of air holes propagating at $1550 \mathrm{~nm}$ wavelength. In particular, our design allows an easy molding of the triangular array of air holes by NIL. We theoretically confirmed that the resulting band gap and the line defect modes of the polymer slabs can also exist in structures with the designed parameters. Furthermore, we implemented them in the air-suspended membrane-type 2D PPhCS waveguides.

As a material for implementing 2D PPhCS waveguides, we used polystyrene with a refractive index of 1.59. To obtain a PBG as broad as possible in the slab with air holes, we chose the air bridge structure with triangular lattice patterns. Before starting the slab design, we deliberated the proper parameters to easily mold the air hole structure by NIL. For instance, to avoid damage of structures during demolding, structures with a small air-fill factor (area covered by holes), i.e., by using stamps with a small pillar diameter, are easier to mold than large ones. In the same way, sagging is reduced if the perforated membrane is more robust. The gap between air holes must be more than $0.4 a$ ( $a$ is the lattice constant), which makes the PBG of the polymer slab with air holes narrow. Large arrays of dense pillars are more difficult to demold than small arrays, due to the accumulation of high local demolding forces. Often this can be facilitated by using a higher initial resist thickness, resulting in a high residual layer, which reduces the risk of resist detachment from the substrate.

The commercial BANDSOLVE simulator can carry out the 3D calculations of the photonic band structure for a unit cell and a supercell. Figure 1(a) shows the photonic band structure for TE-like modes (even parity) of the polystyrene PhCS waveguides suspended in air with a triangular array of air holes when the hole radius $r=0.3 a$ and the slab thickness $t$ $=1.0 \mathrm{a}$. We see that the polystyrene PhCS can exhibit a complete in-plane PBG between 0.486 and 0.5 . The band gap is so narrow due to the low refractive index of the polymer. We set the center wavelength of the PBG at $1550 \mathrm{~nm}$ wave- 

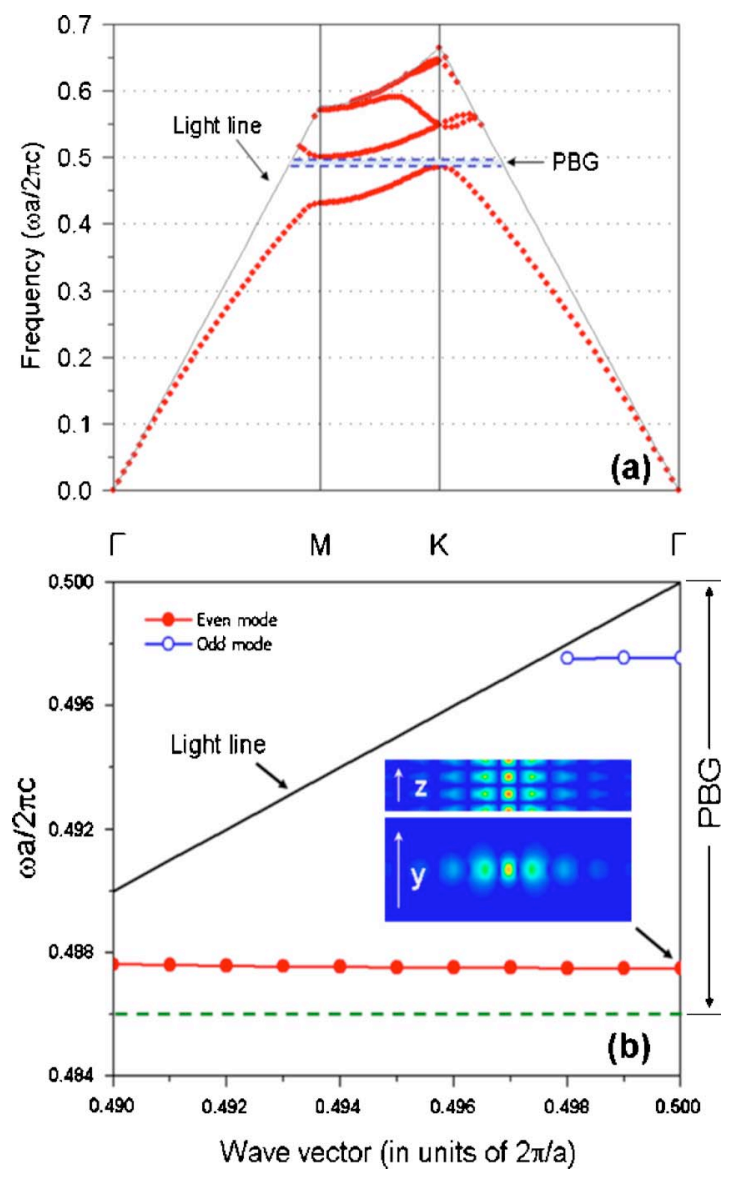

FIG. 1. (Color online) (a) Photonic band structure for the TE-like modes (even parity) of polystyrene PhCS waveguides suspended in air with a triangular array of air holes $[r=0.3 a, t=1.0 a$, where $a$ is the lattice constant (period), $r$ is the hole radius, and $t$ is the slab thickness]. The frequency is normalized to $2 \pi c / a$, where $c$ is the light velocity in vacuum. (b) Photonic bands of defect modes for the width of line defect $d=0.9 \mathrm{~W}$, where $W$ is the width of the line defect created by removing a line of holes in the $\Gamma K$ direction. The closed (open) circles denote the even (odd) defect modes that are symmetric (asymmetric) in the plane bisecting the line defect.

length, and thus the lattice constant $(a)$ of the triangular array is $764 \mathrm{~nm}$, the diameter of hole $2 R=458.5 \mathrm{~nm}(0.6 a)$, and the slab thickness $t=764 \mathrm{~nm}(1.0 a)$. Figure 1(b) shows the photonic bands for the width of line defect $d=0.9 \mathrm{~W}$, where $W$ is the width of the line defect, created by removing a line of holes in the $\Gamma K$ direction. The inset shows the field distribution of even modes at $k=0.5(2 \pi / a)$, where $z$ is the propagating direction and $y$ is the vertical one. Even in the case of designed parameters $(r=0.3 a, t=1.0 a$, and $d=0.9 \mathrm{~W})$, we could see that the field is strongly localized around the line defect, indicating creation of guided modes propagating along the line defect. However, the bands of even/odd modes are so flat that the group velocities are extremely small. This means that the guided range is narrow and the transit time per unit transmission length is large, and as a result the optical power dissipates. ${ }^{12}$ These characteristics of the defect modes have already been experimentally observed in the SOI PhCS waveguides. ${ }^{13}$

A large-area uniform silicon stamp with circular dot structures for the replication of 2D PhCS waveguides was fabricated by using electron-beam lithography and inductively coupled plasma etching. The diameter of the silicon pillars is about $460 \mathrm{~nm}$ and the height about $880 \mathrm{~nm}$. We applied the nanoimprint lithography process to replicate $2 \mathrm{D}$

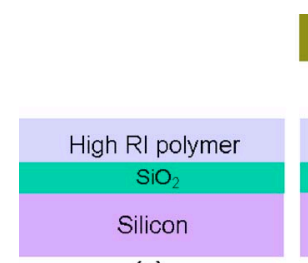

(a)

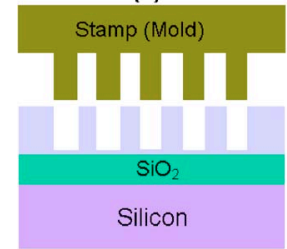

(d)

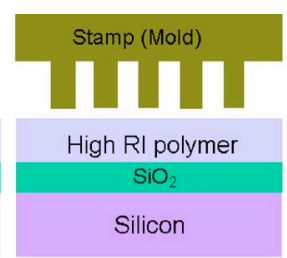

(b)

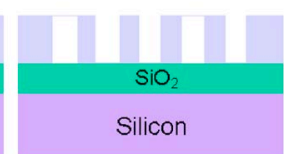

(e)

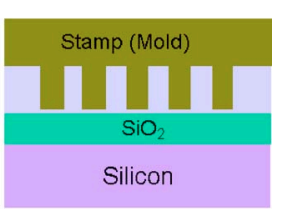

(c)

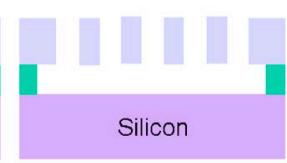

(f)
FIG. 2. (Color online) Fabrication process schematics of nanoimprint lithography for the air-suspended membrane-type 2D PPhCS waveguide.

periodic patterns on polystyrene thin films. Figure 2 shows the process schematics for the fabrication of the airsuspended membrane-type 2D PPhCS waveguides. The polystyrene film coated substrate was imprinted for $20 \mathrm{~min}$ at $180^{\circ} \mathrm{C}$ by applying a pressure of 30 bars. The average depth of imprinted holes is about $850 \mathrm{~nm}$ which is a little bit lower than the height of the stamp. The residual slab thickness underneath the air hole patterns is about $30 \mathrm{~nm}$, which was found to be sufficient to obtain a fine replication of the densely packed photonic crystal nanostructures. An anisotropic $\mathrm{O}_{2}$ plasma etching step was used to open hole windows at the bottom to expose the substrate to the following wet etching step. The air-suspended membrane-type 2D polystyrene PhCS waveguides were realized by selectively removing the $\mathrm{SiO}_{2}$ substrate underneath the triangular array of air holes. A buffered oxide etch was used for $15 \mathrm{~min}$ to generate a gap of about $1 \mu \mathrm{m}$ (the thickness of the $\mathrm{SiO}_{2}$ layer). As shown in Fig. 3 (the structure was cleaved after the release of the membrane), the perforated membrane spans over the whole area $\left(300 \times 30 \mu \mathrm{m}^{2}\right)$ without touching the bottom of the substrate. The diameter of the air holes and the lattice constant are 454 and $760 \mathrm{~nm}$, respectively, which are

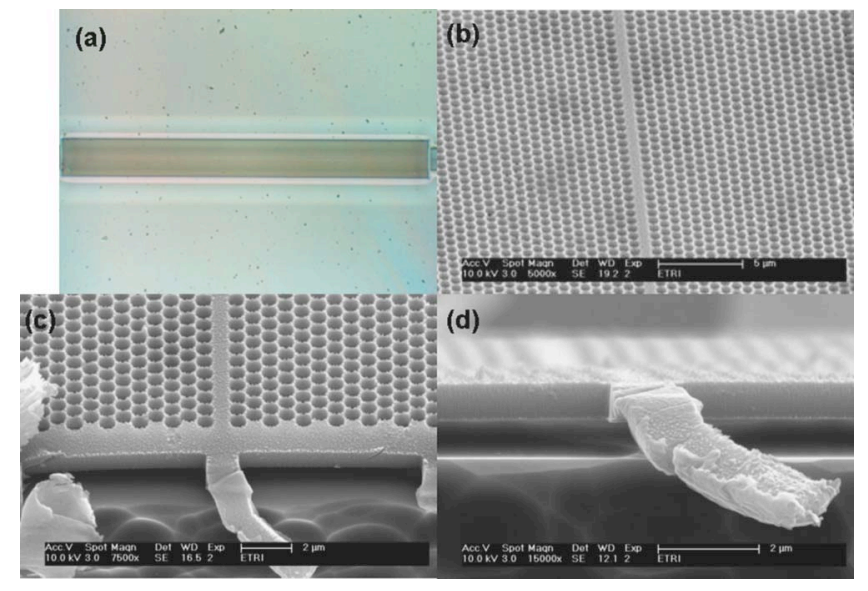

FIG. 3. (Color online) Field emission scanning electron microscopy images of the large-area 2D polystyrene photonic crystal slabs with a triangular array of air holes and a line defected waveguide structure fabricated by NIL; (a) top view of the $300 \times 30 \mu \mathrm{m}^{2}$ large array, and [(b)-(d)] different side views. (c) and (d) show a conventional waveguide at the end of the PhCS waveguide. The triangular array of air holes consists of holes with a diameter of $454 \mathrm{~nm}$ and depth of $738 \mathrm{~nm}$. 


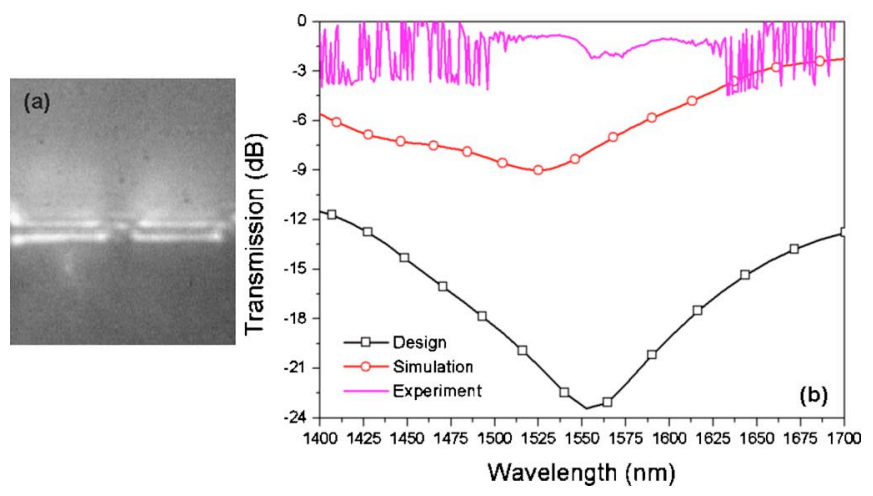

FIG. 4. (Color online) (a) Near field image obtained with a white light source. (b) Optical transmission spectra of a PhCS waveguide for three cases: a simulated PhCS waveguide structure, a structure with the fabricated dimensions, and the measurement of the polystyrene PhCS waveguide fabricated by NIL. All three spectra show the characteristic PBG dip near $1550 \mathrm{~nm}$.

a little smaller than those of the designed parameter. The final depth of the air holes is $738 \mathrm{~nm}$.

Optical measurements have been performed to obtain transmission characteristics. Lensed fibers with a mode size of about $3 \mu \mathrm{m}$ at $1550 \mathrm{~nm}$ were used to couple light into one side of the fabricated PhCS waveguide and to collect the light at the other side. In spite of our efforts to align the fiber to the line defect waveguide formed in the PhCS waveguides, we could not efficiently couple a TE-polarized light into the line defect waveguide. This might be a result of the imperfection of the input/output coupling waveguides at the end of the line defect waveguides, as shown in Figs. 3(c) and 3(d). However, we believe that an efficient coupling to the line defect waveguide will be possible by improving the design of the input/output coupling waveguide. So, in order to confirm a PBG of the PhCS waveguides alone, we shifted the chip to the slab waveguide next to the line defect waveguide without moving the lensed fibers. Figure 4 shows the near field image obtained with a white light source and the optical transmission spectra for a designed PhCS waveguide structure, a simulated PhCS waveguide structure with fabricated dimensions, and a fabricated PhCS waveguide structure. Both the simulations of the designed and fabricated waveguides show the characteristic transmission dip of a PhCS waveguide. This dip, although not as pronounced as in the simulated transmission characteristic, is clearly visible in the center of the flat transmission characteristics of the fabricated device and is a clear sign of a PBG. The transmission spectrum for the fabricated PhCS waveguide structure was obtained by subtracting the spectrum of fiber-fiber coupling (without $\mathrm{PhC}$ ) from that of fiber-PhC-fiber coupling. Therefore, even though the dip in transmission close to $1550 \mathrm{~nm}$ was shallow $(-1.5 \mathrm{~dB})$, we believe that it is a stop band in the $\Gamma K$ direction. It is considered that the seemingly disappointing shallow dip is because an uncoupled light at an input section, which is caused by the large mode mismatch between the lensed fiber and the $\mathrm{PhC}$ device in the vertical direction, was largely coupled into the output lensed fiber and affected the transmission spectrum. In addition, the dis- tance between the output lensed fiber and the input lensed fiber was very short because the $\mathrm{PhC}$ device was very small. For those reasons, the stop band is likely to be shallow. Compared to the simulated prediction, the optical transmission characteristics of the fabricated air-suspended membranetype 2D polystyrene PhCS waveguides are shifted to the longer wavelength region. This is most likely to be the consequence of the fabrication and material tolerances, particularly the uncertainty of the exact refractive index of polystyrene at $1550 \mathrm{~nm}$ wavelength and the inaccuracy of the air hole diameter and slab thickness of the fabricated PhCS waveguides.

With the aid of an innovative NIL process, the airsuspended membrane-type $2 \mathrm{D}$ polystyrene $\mathrm{PhCS}$ waveguides were fabricated. The fabricated $2 \mathrm{D}$ polystyrene PhCS showed a PBG in the $1550 \mathrm{~nm}$ wavelength range. Optical transmission measurements were compared with theory, showing reasonably good agreement. It is expected that by optimization of the input/output fiber coupling structures, defect waveguides can be fabricated and measured. Furthermore by using polymer materials with higher refractive index, a PBG with more pronounced characteristics can be achieved. NIL is likely to be an attractive technology to rapidly and inexpensively implement large-area, ultracompact photonic crystal integrated circuits using relatively simple and straightforward fabrication steps.

This research was supported by the Ministry of Science and Technology through the Korea-Switzerland International Cooperative Research Program and 21st Century Frontier Research Program (Center for Nanoscale Mechatronics and Manufacturing). Many thanks are due to Sandro Bellini and Konrad Vogelsang (PSI Villigen) for their contributions to process optimization.

${ }^{1}$ J. D. Joannopoulos, R. D. Meade, and J. N. Winn, Photonic Crystals: Molding the Flow of Light (Princeton University Press, Princeton, 1995), Chap. 4, p. 40.

${ }^{2}$ S. Noda, M. Imada, M. Okano, S. Ogawa, M. Mochizuki, and A. Chutinan, IEEE J. Quantum Electron. 38, 726 (2002).

${ }^{3}$ E. Chomski and G. A. Ozin, Adv. Mater. (Weinheim, Ger.) 12, 1071 (2000).

${ }^{4}$ C. J. M. Smith, R. M. De La Rue, M. Rattier, S. Olivier, H. Benisty, C. Weisbush, T. F. Krauss, R. Huodrè, and U. Osterle, Appl. Phys. Lett. 78, 1487 (2001).

${ }^{5}$ J. D. Joannopoulos, P. R. Villeneuve, and S. Fan, Nature (London) 386, 143 (1997).

${ }^{6}$ L. Guo and J. Jay, J. Phys. D 37, R123 (2004).

${ }^{7}$ M. De Vittorio, M. T. Todaro, T. Stomeo, R. Cingolani, D. Cojoc, and E. Di Fabrizio, Microelectron. Eng. 73, 388 (2004).

${ }^{8}$ H. Schift, S. Park, B. Jung, C.-G. Choi, C.-S. Kee, S.-P. Han, K.-B. Yoon, and J. Gobrecht, Nanotechnology 16, S261 (2005).

${ }^{9}$ C. Liguda, G. Bottger, A. Kuligk, R. Blum, M. Eich, H. Roth, J. Kunert, W. Morgenroth, H. Elsner, and H. G. Meyer, Appl. Phys. Lett. 78, 2434 (2001).

${ }^{10}$ C.-S. Kee, S. P. Han, K. B. Yoon, C.-G. Choi, H. K. Sung, S. S. Oh, H. Y. Park, S. Park, and H. Schift, Appl. Phys. Lett. 86, 51101 (2005).

${ }^{11}$ H. Schift, S. Bellini, U. Pieles, and J. Gobrecht, J. Microlithogr., Microfabr., Microsyst. 5, 011010 (2006).

${ }^{12}$ M. Notomi, A. Shinya, K. Yamada, J. Takahashi, C. Takahashi, and I. Yokohama, IEEE J. Quantum Electron. 38, 736 (2002).

${ }^{13}$ K. Yamada, H. Morita, A. Shinya, and M. Notomi, Opt. Commun. 198, 395 (2001). 